\title{
Simultaneous determination of toluene and xylene metabolites in urine by gas chromatography
}

\author{
J. R. CAPEROS AND J. G. FERNÁNDEZ \\ From the Occupational Health and Industrial Hygiene Service, Department of Chemistry, University \\ of Neuchâtel, Bellevaux 51, 2000 Neuchâtel, Switzerland
}

\begin{abstract}
A gas chromatographic method for simultaneous determination of hippuric and o-, $\mathrm{m}$-, and p-methylhippuric acids (metabolites of toluene and xylene) in urine is described. The analytical procedure is based on the extraction of the aromatic metabolites with ethyl acetate containing the internal standard and on a methylation with 3-methyl-1-p-tolyltriazene. With this method, which does not require much time and handling, the different acids can be satisfactorily determined with high sensitivity and specificity. A statistical study shows a good reproducibility for the determination of hippuric and o-, $\mathrm{m}$-, and p-methylhippuric acids. The coefficient of variation for 10 determinations in all cases was less than $5 \%$.
\end{abstract}

Toluene and xylene are the principal homologues of benzene and may replace it in many industrial operations. The carbonisation of coal was for a long time the major source of these two solvents, but today they are obtained mainly from petroleum and by petrochemical processes.

Toluene and xylene may be encountered as relatively pure substances or as constituents of solvent mixtures. Occupational exposure may occur from transfer of liquid, spillage or from leaking equipment. There is also a high risk of exposure when toluene or xylene are present as components of paints, thinners, lacquers or as solvents for gums, fats and resins.

In the body, toluene is mainly oxidised into benzoic acid which after conjugation with glycine is eliminated as hippuric acid, in the urine (Quick, 1931). Benzoic acid, which is a normal urinary constituent occurring naturally or derived from food containing benzoate added as a preservative, is also formed from phenylalanine (Meister, 1965) and other alkylbenzenes (El Masry et al., 1956). Quantitative aspects of hippuric acid formation are of interest not only as a test of industrial exposure to toluene but also in the evaluation of liver function (Quick test), in the study of pharmacokinetics of salicylic acid (Amsel and Levy, 1969; Levy et al., 1969) and to observe patients with the Lesch-Nyhan syndrome (Jolley and Scott, 1970; Beardmore and Kelley, 1971).

Received for publication 14 February 1977 Accepted for publication 5 April 1977
The commercial product of xylene is a blend of three isomers, the largest proportion consisting of the meta-compound (up to $75-80 \%$ ) and the smallest of the para-compound (up to $5 \%$ ). After absorption, xylenes are mainly metabolised into toluic acid which, when conjugated with glycine or glucuronic acid give the corresponding ortho-, meta- and paramethylhippuric or toluylglucuronic acids. Only a small percentage is eliminated by pulmonary excretion and by nuclear oxidation with formation of xylenols.

Until now, different methods, including colorimetry (Gaffney et al., 1954; Ogata et al., 1962; Umberger and Fioresse, 1963), ultraviolet spectrophotometry (Pagnotto and Lieberman, 1967; Ikeda and Ohtsuji, 1969) and gas chromatography (Šedivec and Flek, 1970; Buchet and Lauwerys, 1973; Engström et al., 1976), were used to determine the urinary metabolites of toluene and xylene. Among the various analytical methods proposed, the gas chromatography technique provides the greatest efficiency and easiest determination. Colorimetric and direct UV-spectrophotometric methods are subject to interference from a wide variety of compounds. Removal of this interference is tedious and in many cases incomplete.

The purpose of this paper is to describe a new specific and sensitive gas liquid chromatography procedure for the simultaneous determination of hippuric and o-, m-, and p-methylhippuric acids for monitoring workers exposed occupationally to toluene and/or xylene. This method is based on the 
formation of methyl esters of hippuric and o-, $\mathrm{m}-$, and p-methylhippuric acids. For the methylation process of these acids, 3-methyl-1-p-tolyltriazene is used, which reacts directly and instantaneously with carboxylic acids (White et al., 1968; Humbert and Fernández, 1976; Caperos and Fernández, 1976). Furthermore, the use of an internal standard (tridecanoic acid) gives the method a very good reproducibility. During the last few years, this procedure has been regularly used in our laboratory and proved to be satisfactory.

\section{Materials and methods}

\section{CHEMICALS}

Ethyl acetate, chloroform, methanol, hydrochloric acid and sulphuric acid were purchased from Merck (Darmstadt, Germany). Hippuric acid, tridecanoic acid, o-, and m-methylbenzoic acids, p-methylbenzoyl chloride, glycine and 3-methyl-1-p-tolyltriazene were furnished by Fluka (Buchs, Switzerland). $\mathrm{O}-$, and m-methylbenzoyl chloride were synthesised from o-, and m-methylbenzoic acids respectively, following Vogel's (1967) procedure for aromatic chlorides. O-, m-, and p-methylhippuric acids were prepared from o-, m-, and p-methylbenzoyl chloride and glycine, using Vogel's (1967) method for hippuric acid synthesis. The o-, m-, and p-methylhippuric acids were converted to the corresponding methyl esters using methanol with concentrated sulphuric acid as catalyst.

For identification of these compounds infrared and nuclear magnetic resonance analyses were conducted and their purity was determined by gas chromatography. 3-methyl-1-p-tolyltriazene was purified by sublimation and recrystallisation in hexane before use (White et al., 1968).

\section{APPARATUS}

A Perkin-Elmer model 900 gas chromatograph equipped with glass column $(450 \mathrm{~cm}$ long, $2 \mathrm{~mm}$ internal diameter) packed with Chromosorb G (AW-DMCS, 80-100 mesh) coated with 4\% Apiezon $M$ was used. $\mathrm{N}_{2}$ was the carrier gas $(20 \mathrm{ml} / \mathrm{min})$ and a hydrogen flame-ionisation detector unit was employed with a hydrogen flow of $60 \mathrm{ml} / \mathrm{min}$ and an air flow of $450 \mathrm{ml} / \mathrm{min}$. The oven was maintained at a temperature of $220^{\circ} \mathrm{C}$, the manifold and injector at $250^{\circ} \mathrm{C}$. Chromatograms were obtained on a Perkin-Elmer recorder model 56 using a chart speed of $5 \mathrm{~mm} / \mathrm{min}$.

The determination of the MS spectra was carried out with a Perkin-Elmer model 990 gas chromatograph coupled with a Hitachi mass spectrometer RMU-6L, under the following conditions: ionisation voltage $68.6 \mathrm{eV}$, ion source temperature $205^{\circ} \mathrm{C}$, ion accelerative voltage $500 \mathrm{eV}$, inlet vapour temperature $215^{\circ} \mathrm{C}$.

\section{URINE TREATMENT}

$0.5 \mathrm{ml}$ of urine were transferred intoaglass stoppered tube and acidified with $0.1 \mathrm{ml}$ of $10 \mathrm{~mol} / \mathrm{l} . \mathrm{HCl}$ After introduction of $5 \mathrm{ml}$ of internal standard solution (tridecanoic acid, $25 \mathrm{mg} / \mathrm{l}$, in ethyl acetate), the tube was shaken for 5 minutes, $3 \mathrm{ml}$ of the ethyl acetate extract (containing o-, m-, p-methylhippuric, hippuric and tridecanoic acids) were added to $2 \mathrm{ml}$ of the methylating solution (3-methyl1-p-tolyltriazene in ethyl acetate, $36 \mathrm{~g} / \mathrm{l}$ ) in a crystallising dish and this mixture was evaporated slowly to dryness at a temperature lower than $50^{\circ}$ C. After cooling the methylated sample was dissolved in $0.2 \mathrm{ml}$ of chloroform. The excess of methylating reagent was removed by shaking the chloroform solution with $1 \mathrm{ml}$ of $3 \mathrm{~mol} / 1 \mathrm{HCl}$ which produced the water-soluble chlorohydrate. Finally, $2 \mu \mathrm{l}$ of the organic phase were injected into the gas chromatograph for analysis.

\section{DEGREE OF METHYLATION}

To investigate the efficiency of methylation, known concentrations of hippuric, o-, m-, and p-methylhippuric acid methyl esters were prepared by diluting these esters in ethyl acetate. $1 \mathrm{ml}$ of ethyl acetate containing $100 \mathrm{mg} / \mathrm{l}$ of tridecanoic acid methyl ester (internal standard) was added to $3 \mathrm{ml}$ of the standard solution. The mixture was evaporated to dryness at a temperature not higher than $50^{\circ} \mathrm{C}$. The residue was dissolved in chloroform and $2 \mu \mathrm{l}$ were then analysed by gas liquid chromatography. Calibration curves were calculated (Figures 1 and 2). These data were then compared to results obtained from standard solutions of hippuric acid and o-, m-, and

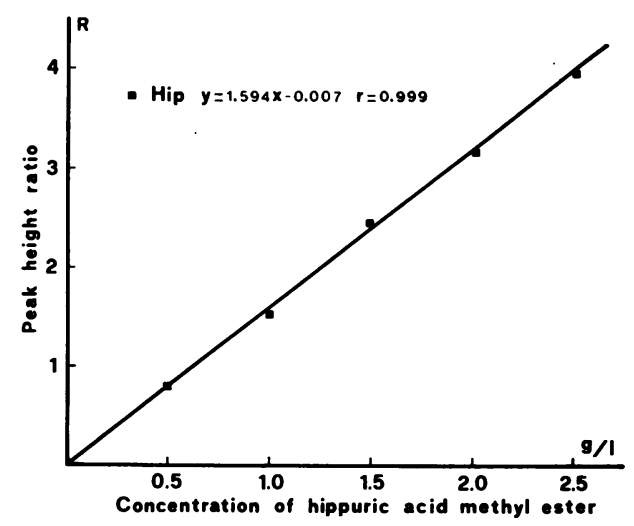

Fig. 1 Standard curve of hippuric acid methyl ester in ethyl acetate. 


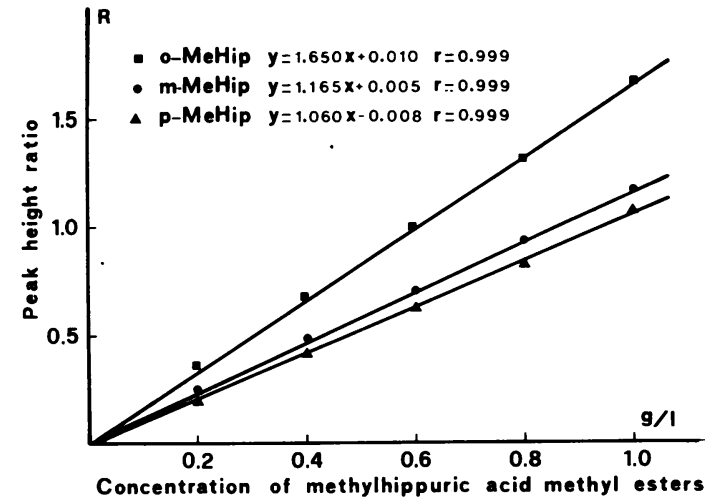

Fig. 2 Standard curves of $o-, m$-, and p-methylhippuric acid methyl esters in ethyl acetate.

p-methylhippuric acid in ethyl acetate and methylated with 3-methyl-1-p-tolyltriazene (Table 1).

DEGREE OF METHYLATION AND EXTRACTION The total degree of both methylation and extraction processes was studied as follows. Known concentrations of hippuric o-, m-, and p-methylhippuric acids were prepared by diluting these acids in water or in urine. $5 \mathrm{ml}$ of each solution were treated as described under 'urine treatment' with these variations: the solvent of extraction did not contain the internal standard (tridecanoic acid), but its methyl ester was added to the ethyl acetate extract later. The ratio of the peak heights of the hippuric, and o-, m-, and p-methylhippuric acids to the internal standard was calculated (Tables 2 and 3) and compared with the peak height ratios between hippuric, and o-, m-, and p-methylhippuric acid methyl esters and the internal standard shown in Figures 1 and 2.

\section{Results}

Figure 3 shows a characteristic gas chromatogram of the treated urine from a worker exposed to vapours of toluene and xylene in a printing office. The five acid methyl esters were well separated and eluted within 20 minutes. All the aromatic acids studied

Table 1 Study of methylation. Reproducibility and recovery of the analyses of metabolites in ethyl acetate

\begin{tabular}{|c|c|c|c|c|c|}
\hline Metabolites & $\begin{array}{l}\text { Concentration of } \\
\text { acids }(\mathrm{g} / \mathrm{l})\end{array}$ & No. of analyses & $\begin{array}{l}\text { Mean of peak } \\
\text { height ratios }\end{array}$ & $\begin{array}{l}\text { Coefficient of } \\
\text { variation }(\%)\end{array}$ & $\begin{array}{l}R \\
(\%)\end{array}$ \\
\hline Hippuric acid & $2 \cdot 5$ & 10 & 3.95 & $4 \cdot 0$ & $99 \cdot 6$ \\
\hline Hippuric acid & 1.5 & 10 & 2.47 & $2 \cdot 8$ & 96.8 \\
\hline Hippuric acid & 1.0 & 10 & 1.52 & 3.4 & 95.8 \\
\hline o-methylhippuric acid & 1.0 & 10 & 1.69 & 1.4 & 98.1 \\
\hline o-methylhippuric acid & 0.6 & 10 & 0.99 & $3 \cdot 1$ & $98 \cdot 3$ \\
\hline o-methylhippuric acid & 0.4 & 10 & 0.67 & $3 \cdot 1$ & $97 \cdot 3$ \\
\hline m-methylhippuric acid & $1 \cdot 0$ & 10 & $1 \cdot 16$ & 3.5 & $100 \cdot 0$ \\
\hline m-methylhippuric acid & 0.4 & 10 & 0.47 & 2.4 & $100 \cdot 0$ \\
\hline p-methylhippuric acid & $1 \cdot 0$ & 10 & 1.05 & 6.0 & $100 \cdot 0$ \\
\hline p-methylhippuric acid & 0.6 & 10 & 0.63 & $3 \cdot 5$ & $100 \cdot 0$ \\
\hline p-methylhippuric acid & 0.4 & 10 & 0.41 & $2 \cdot 5$ & $100 \cdot 0$ \\
\hline
\end{tabular}

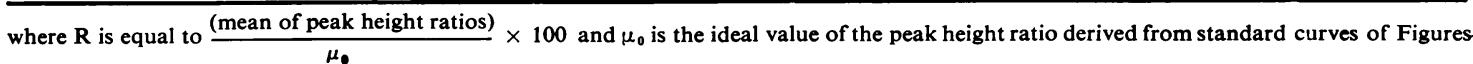
1 and 2.

Table 2 Study of methylation and extraction. Reproducibility and recovery of the analyses of metabolites in water

\begin{tabular}{|c|c|c|c|c|c|}
\hline Metabolites & $\begin{array}{l}\text { Concentration of } \\
\text { acids }(g / l)\end{array}$ & No. of analyses & $\begin{array}{l}\text { Mean of peak } \\
\text { height ratios }\end{array}$ & $\begin{array}{l}\text { Coefficient of } \\
\text { variation }(\%)\end{array}$ & $\begin{array}{l}R \\
(\%)\end{array}$ \\
\hline $\begin{array}{l}\text { Hippuric acid } \\
\text { Hippuric acid } \\
\text { Hippuric acid } \\
\text { o-methylhippuric acid } \\
\text { o-methylhippuric acid } \\
\text { o-methylhippuric acid } \\
\text { m-methylhippuric acid } \\
\text { m-methylhippuric acid } \\
\text { m-methylhippuric acid } \\
\text { p-methylhippuric acid } \\
\text { p-methylhippuric acid } \\
\text { p-methylhippuric acid }\end{array}$ & $\begin{array}{l}2.5 \\
1.5 \\
0.5 \\
1.0 \\
0.6 \\
0.4 \\
1.0 \\
0.6 \\
0.4 \\
1.0 \\
0.6 \\
0.4\end{array}$ & $\begin{array}{l}10 \\
10 \\
10 \\
10 \\
10 \\
10 \\
10 \\
10 \\
10 \\
10 \\
10 \\
10\end{array}$ & $\begin{array}{l}3.35 \\
2.08 \\
0.67 \\
1.42 \\
0.84 \\
0.56 \\
0.99 \\
0.60 \\
0.41 \\
0.90 \\
0.52 \\
0.36\end{array}$ & $\begin{array}{l}2 \cdot 1 \\
2 \cdot 2 \\
1 \cdot 5 \\
2 \cdot 5 \\
4 \cdot 2 \\
1 \cdot 8 \\
2 \cdot 3 \\
2 \cdot 1 \\
4 \cdot 3 \\
1 \cdot 7 \\
4 \cdot 8 \\
3 \cdot 5\end{array}$ & $\begin{array}{l}81 \cdot 3 \\
75 \cdot 5 \\
81 \cdot 9 \\
82 \cdot 4 \\
80 \cdot 0 \\
79 \cdot 8 \\
82 \cdot 4 \\
82 \cdot 4 \\
82 \cdot 4 \\
83 \cdot 7 \\
80 \cdot 0 \\
82 \cdot 4\end{array}$ \\
\hline
\end{tabular}

where $\mathbf{R}$ is equal to $\frac{\text { (mean of peak height ratios) }}{\mu_{0}} \times 100$ and $\mu_{0}$ is the ideal value of the peak height ratio derived from standard curves of Figures 1 and 2. 
Table 3 Study of methylation and extraction. Reproducibility and recovery of the analyses of metabolites in urine

\begin{tabular}{|c|c|c|c|c|c|}
\hline Metabolites & $\begin{array}{l}\text { Concentration of } \\
\text { acids }(\mathrm{g} / \mathrm{l})\end{array}$ & No. of analyses & $\begin{array}{l}\text { Mean of peak } \\
\text { height ratios }\end{array}$ & $\begin{array}{l}\text { Coefficient of } \\
\text { variation }(\%)\end{array}$ & $\begin{array}{l}R \\
(\%)\end{array}$ \\
\hline Hippuric acid & $2 \cdot 5$ & 10 & $4 \cdot 50$ & 2.4 & - \\
\hline Hippuric acid & $2 \cdot 0$ & 10 & 3.81 & $1 \cdot 5$ & - \\
\hline Hippuric acid & $1 \cdot 0$ & 10 & 2.46 & $2 \cdot 2$ & - \\
\hline o-methylhippuric acid & $1 \cdot 0$ & 10 & 1.40 & 1.8 & $82 \cdot 4$ \\
\hline o-methylhippuric acid & 0.6 & 10 & 0.85 & 1.8 & $82 \cdot 4$ \\
\hline o-methylhippuric acid & 0.4 & 10 & 0.55 & $2 \cdot 8$ & 79.8 \\
\hline m-methylhippuric acid & $1 \cdot 0$ & 10 & 1.00 & $1 \cdot 5$ & $81 \cdot 0$ \\
\hline m-methylhippuric acid & 0.6 & 10 & 0.60 & 2.5 & $80 \cdot 0$ \\
\hline m-methylhippuric acid & 0.4 & 10 & 0.42 & 3.6 & $85 \cdot 7$ \\
\hline p-methylhippuric acid & $1 \cdot 0$ & 10 & 0.94 & $2 \cdot 7$ & 86.4 \\
\hline p-methylhippuric acid & 0.6 & 10 & 0.55 & 1.8 & 82.4 \\
\hline p-methylhippuric acid & 0.4 & 10 & 0.37 & $2 \cdot 3$ & 85.6 \\
\hline
\end{tabular}

where $R$ is equal to (mean of peak height ratios) $\times 100$ and $\mu_{0}$ is the ideal value of the peak height ratio derived from standard curves of Figures 1 and 2.

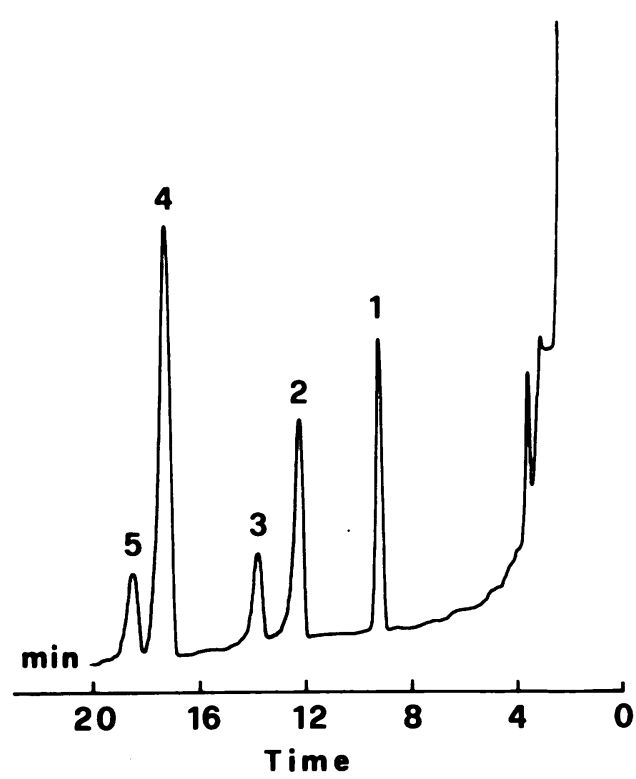

Fig. 3 Chromatogram obtained by analysing the urine of a worker exposed to xylene vapours in a printing office. Methyl esters of tridecanoic acid (1), of hippuric acid (2) and o-, m-, p-methylhippuric acids $(3,4,5)$.

were identified by comparing their retention behaviour and their mass spectra with reference compounds.

An analytical curve was obtained by subjecting the reference compounds to all steps of analysis and by plotting the peak height ratio between each acid studied and the internal standard versus concentrations. Standard solutions of hippuric acid from 0.5 to $2.5 \mathrm{~g} / 1$ and o-, m- and p-methylhippuric acids

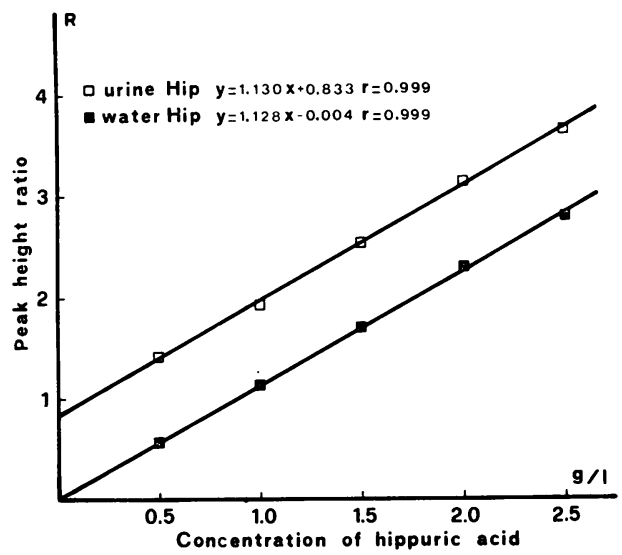

Fig. 4 Standard curve of hippuric acid methyl ester in water and in urine.

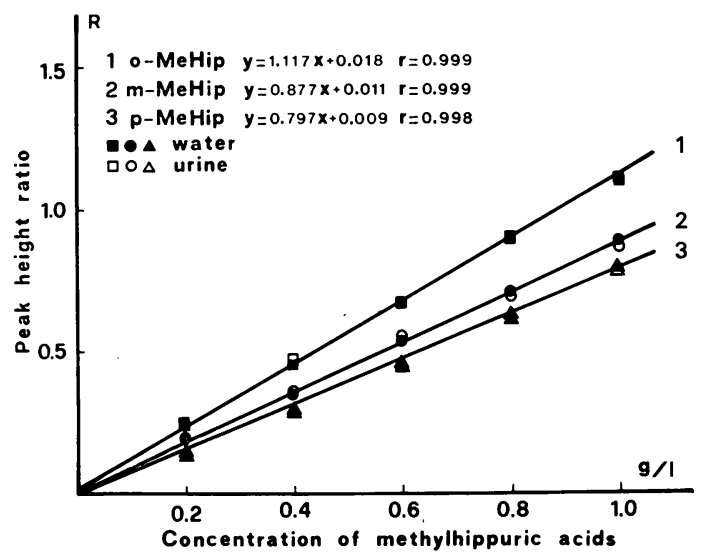

Fig. 5 Standard curves of o-, $m$-, and p-methylhippuric acids in water and in urine. 
from 0.2 to $1.0 \mathrm{~g} / \mathrm{l}$ were prepared both in water and in urine. Calibration curves obtained from these solutions are shown in Figures 4 and 5. As urine already contains some hippuric acid, the calibration curve is separated from the water calibration curve, resulting in two parallel lines.

The esterification of hippuric, o-, m-, and pmethylhippuric acids is rapidly carried out and the yield of this reaction is constant (mean $98.7 \pm 1.5 \%$ ) as shown in Table 1 . The total yield of both methylation and extraction procedures must be constant to ensure good reproducibility of this method: our results, shown in Tables 2 and 3, confirm that both processes give constant yields (mean $81.2 \pm 2.2 \%$ in water, $82.9 \pm 2.5 \%$ in urine). The yield of extraction depends on solubility of the hippuric, o-, m-, and p-methylhippuric acids in water, urine or ethyl acetate, and is modified when the volume of two phases (organic or aqueous) is changed. Like Buchet and Lauwerys (1973), we found that the degree of extraction of the four methyl derivatives from the urine with ethyl acetate was constant over $\mathrm{HCl}$ concentrations ranging between $0.05 \mathrm{~mol} / \mathrm{l}$ and $10 \mathrm{~mol} / \mathrm{l}$.

\section{Conclusion}

This work describes a gas chromatographic method which allows the determination in the urine of hippuric, and o-, m-, and p-methylhippuric acids, metabolites of toluene and xylene. The use of tridecanoic acid as internal standard and that of a rapid and efficient methylation with 3-methyl-1-ptolyltriazene make this analysis simple, accurate and reproducible.

The best resolution of methyl ester derivatives is obtained by using a $4 \%$ Apiezon $M$ filled column while a FID detector allows high sensitivity.

The smallest amount of these acids in urine distinguishable by our procedure was $5 \mathrm{mg} / \mathrm{l}$, but with larger quantities of sample a considerably higher sensitivity may be achieved.

By means of this method it is possible to determine from the same urinary sample the different aromatic acids, metabolites of toluene and xylene, with high specificity in a relatively short time. The method can therefore be used for the occupational monitoring of exposure to toluene and xylene, as well as for studies in industrial toxicology.

We are grateful to the Fonds National Suisse pour la Recherche Scientifique for financial support. Thanks are due to B. E. Humbert for skilful technical assistance.

\section{References}

Amsel, L. P., and Levy, G. (1969). Drug biotransformation interactions in man. II. A pharmacokinetic study of the simultaneous conjugation of benzoic and salicylic acids with glycine. Journal of Pharmaceutical Science, 58, 321-326.

Beardmore, T. D., and Kelley, W. N. (1971). Ultravioletabsorbing compounds in urine from patients with hereditary disorders of purine and pyrimidine metabolism. Clinical Chemistry, 17, 795-801.

Buchet, J. P., and Lauwerys, R. R. (1973). Measurement of urinary hippuric and m-methylhippuric acids by gas chromatography. British Journal of Industrial Medicine 30, 125-128.

Caperos, J. R., and Fernández, J. G. (1976). Dosage des acides mandélique et phénylglyoxylique dans l'urine par chromatographie en phase gazeuse. Archives des maladies professionnelles de Médecine du Travail et de Sécurité Sociale, 37, 387-391.

El Masry, A. M., Smith, J. N., and Williams, R. T. (1956). Detoxication. LXIX. The metabolism of alkylbenzenes: n-propylbenzene and n-butylbenzene with further observations on ethylbenzene. Biochemistry Journal, 64, 50-56.

Engström, K., Husman, K., and Rantanen, J. (1976). Measurement of toluene and xylene metabolites by gas chromatography. International Archives of Occupational and Environmental Health, 36, 153-160.

Gaffney, G. W., Schreier, K., DiFerrante, N., and Altman, K. I. (1954). The quantitative determination of hippuric acid. Journal of Biological Chemistry, 206, 695-698.

Humbert, B. E., and Fernández, J. G. (1976). Simultaneous determination of trichloracetic acid and trichlorethanol by gas chromatography. International Archives of Occupational and Environmental Health, 36, 235-241.

Ikeda, M., and Ohtsuji, H. (1969). Significance of urinary hippuric acid determination as an index of toluene exposure. British Journal of Industrial Medicine, 26, 244-246.

Jolley, R. L., and Scott, C. D. (1970). Preliminary results from high-resolution analyses of ultraviolet-absorbing and carbohydrate constituents in several pathologic body fluids. Clinical Chemistry, 16, 687-696.

Levy, G., Amsel, L. P., and Elliot, H. C. (1969). Kinetics of salicyluric acid elimination in man. Journal of Pharmaceutical Science, 58, 827-829.

Meister, A. (1965). Biochemistry of the amino acids, 2nd edition. Academic Press: New York.

Ogata, M., Sugiyama, K., and Moriyasu, H. (1962). Studies on poisoning. IV. Toluene concentration in air and urinary hippuric acid measured by paperchromatography and mass screening examination method. Acta Medicinae Okayama, 16, 283-292.

Pagnotto, L. D., and Lieberman, L. M. (1967). Urinary hippuric acid excretion as an index of toluene exposure. American Industrial Hygiene Association Journal, 28, 129-134.

Quick, A. J. (1931). The conjugation of benzoic acid in man. Journal of Biological Chemistry, 92, 65-85.

Sedivec, V., and Flek, J. (1970). Bestimmung toxischer Substanzen und ihrer Metaboliten in Biologischen Flüssigkeiten mittels der Gaschromatographie. V. Hippursäure in Urin. Collection of Czechoslovak Chemical Communications, 35, 3265-3273.

Umberger, J. C., and Fioresse, F. F. (1963). Colorimetric method for hippuric acid. Clinical Chemistry, 1, 91-96.

Vogel, A. L. (1967). A Textbook of Practical Organic Chemistry, 3rd edition. Longmans: London.

White, E. H., Baum, A. A., and Eitel, D. E. (1968). 1-methyl3-p-tolyltriazene and its use in the esterification of acids. Organic Syntheses, 48, 102-105. 